TITLE:

\title{
Quantum disordered ground states in frustrated antiferromagnets with multiple ring exchange interactions
}

\author{
AUTHOR(S): \\ Fujimoto, S
}

CITATION:

Fujimoto, S. Quantum disordered ground states in frustrated antiferromagnets with multiple ring exchange interactions. PHYSICAL REVIEW B 2005, 72(2): 024429.

ISSUE DATE:

2005-07

URL:

http://hdl.handle.net/2433/50021

RIGHT:

Copyright 2005 American Physical Society 
PHYSICAL REVIEW B 72, 024429 (2005)

\title{
Quantum disordered ground states in frustrated antiferromagnets with multiple ring exchange interactions
}

\author{
Satoshi Fujimoto \\ Department of Physics, Kyoto University, Kyoto 606-8502, Japan \\ (Received 27 December 2004; revised manuscript received 28 February 2005; published 14 July 2005)
}

\begin{abstract}
We present a certain class of two-dimensional frustrated quantum Heisenberg spin systems with multiple ring exchange interactions which are rigorously demonstrated to have quantum disordered ground states without magnetic long-range order. The systems considered in this paper are $s=1 / 2$ antiferromagnets on a honeycomb and square lattices, and an $s=1$ antiferromagnet on a triangular lattice. We find that for a particular set of parameter values, the ground state is a short-range resonating valence bond state or a valence bond crystal state. It is shown that these systems are closely related to the quantum dimer model introduced by Rokhsar and Kivelson as an effective low-energy theory for valence bond states.
\end{abstract}

DOI: 10.1103/PhysRevB.72.024429

PACS number(s): 75.10.Jm, 75.40.- s

\section{INTRODUCTION}

Quantum frustrated Heisenberg antiferromagnets have attracted a great deal of interest in connection with the search for exotic phases, such as spin liquids. Anderson proposed a resonating valence bond (RVB) state as a prototype of spin liquids three decades ago. ${ }^{1}$ Since then, a number of works have studied the conjecture that both geometrical frustration and strong quantum fluctuation may destroy magnetic longrange order and stabilize a quantum disordered liquidlike ground state without symmetry breaking. ${ }^{2-17}$ For example, it is believed quite likely that quantum spin systems on Kagome lattices and pyrochlore lattices can exhibit quantum spin liquid states, though the elucidation of their ground states remains an important unsolved problem. In related works, the quantum dimer model (QDM) introduced by Rokhsar and Kivelson has been extensively studied as an effective low-energy theory of quantum disordered states with short-range antiferromagnetic correlation and a spin excitation gap. ${ }^{18-23}$ The important feature of the QDM is that its Hilbert space is spanned by only dimer covering states composed of singlet pairs of nearest-neighbor spins, and this is the key to its success in describing quantum disordered states. ${ }^{18}$ It was shown in the pioneer paper by Rokhsar and Kivelson that a short-range RVB state is realized in the QDM as its exact ground state. It was also argued by several authors that the QDM may be an effective low-energy theory of quantum antiferromagnets on the Kagome lattice. ${ }^{15,23}$

However, it is not clear how to derive the QDM directly from the original quantum spin Hamiltonian by truncating the Hilbert space. Indeed, it appears difficult to find a general answer to this question. Therefore, in this paper, we do not seek such a general result. Instead, to obtain better insight regarding the mechanism stabilizing quantum spin liquids, we attempt to make progress toward determining what kinds of quantum spin systems possess a low-energy sector described by the QDM.

The main result of this paper is the rigorous proof that a certain class of quantum antiferromagnetic spin systems in two dimensions with multiple ring exchange interactions is equivalent to the QDM. These systems exhibit a short-range
RVB state or a valence bond crystal (VBC) state, depending on the values of their parameters. Our results clarify the important role played by the ring exchange interactions in the realization of quantum spin liquid states. ${ }^{24}$

This paper is organized as follows. In Sec. II, we present results of an $s=1 / 2$ Heisenberg spin system with multiple ring exchange interactions defined on a honeycomb lattice. It is shown that for a particular parameter the rigorous ground state is the short-range RVB. In this RVB state, spin-spin correlations decay exponentially, and therefore there is no magnetic long-range order. On the other hand, correlations between spin-singlet dimers exhibit power-law long-distance behavior, implying the existence of low-lying spin-singlet gapless excitations. In Sec. III and Sec. IV, we consider an $s=1 / 2$ model on a square lattice and an $s=1$ model on a triangular lattice, respecticely. Discussion and summary are given in Sec. V.

\section{II. $s=1 / 2$ HEISENBERG ANTIFERROMAGNET ON A HONEYCOMB LATTICE}

\section{A. Model hamiltonian}

We consider the $s=1 / 2$ quantum Heisenberg antiferromagnet on a honeycomb lattice [see Fig. 1(a)], of which the ground state is exactly obtained. The Hamiltonian is given by $H=H_{\mathrm{K}}+H_{\mathrm{R}}$, with
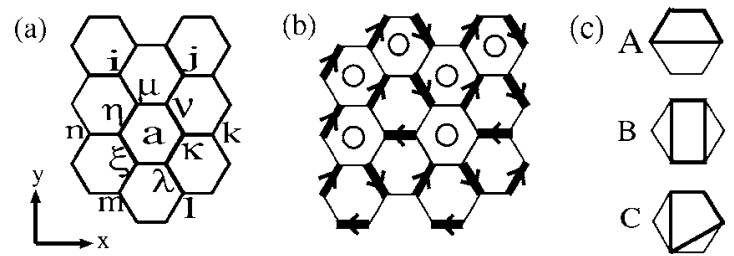

FIG. 1. (a) Honeycomb lattice. (b) Three kinds of four-spin exchange processes on a hexagon. (c) An example of a dimer covering state. The arrows indicate the phase convention of the singlet states. An arrow from $i$ to $j$ represents $O_{i j}|0\rangle$. Hexagons containing circles are covered with unflippable dimers. (d) Staggered VBC. 


$$
\begin{aligned}
H_{\mathrm{K}}= & J_{1}\left[\sum_{(i j)} \boldsymbol{S}_{i} \cdot \boldsymbol{S}_{j}+\frac{1}{2} \sum_{\langle i j\rangle} \boldsymbol{S}_{i} \cdot \boldsymbol{S}_{j}+\frac{3}{8} N\right. \\
& \left.+\frac{2}{5} \sum_{\substack{|j-k|=|j-l||| k-l \mid=\sqrt{3} \\
(i j)(i k)(i l)}}\left(\boldsymbol{S}_{i} \cdot \boldsymbol{S}_{j}\right)\left(\boldsymbol{S}_{k} \cdot \boldsymbol{S}_{l}\right)\right], \\
& H_{\mathrm{R}}=\sum_{a} \mathcal{P}_{a}^{M}\left[J_{2} K_{a}+J_{3} V_{a}\right],
\end{aligned}
$$

$$
\begin{aligned}
K_{a}= & \frac{7}{16} \sum_{(\mu \nu) \in\{a\}} S_{\mu} \cdot S_{\nu}+\frac{1}{8} \sum_{\langle\mu \nu\rangle \in\{a\}} S_{\mu} \cdot S_{\nu}+\frac{1}{8} \sum_{\langle\langle\mu \nu\rangle\rangle \in\{a\}} S_{\mu} \cdot S_{\nu} \\
& -\frac{5}{32}\left[\sum_{A}^{a} P_{4}^{a}+\sum_{B}^{a} P_{4}^{a}-\frac{3}{5} \sum_{C}^{a} P_{4}^{a}\right]+\frac{1}{8} P_{6}^{a}+\frac{13}{64}
\end{aligned}
$$$$
V_{a}=-\frac{1}{16} \sum_{(\mu \nu) \in\{a\}} S_{\mu} \cdot S_{\nu}+\frac{1}{4} \sum_{\substack{(\mu \nu)(\alpha \beta) \in\{a\} \\ \mu<\nu<\alpha<\beta}}\left(\boldsymbol{S}_{\mu} \cdot \boldsymbol{S}_{\nu}\right)\left(\boldsymbol{S}_{\alpha} \cdot \boldsymbol{S}_{\beta}\right)
$$$$
-\sum_{\substack{(\kappa \lambda)(\varepsilon \gamma)(\eta \xi) \in\{a\} \\(\lambda \varepsilon)(\gamma \eta)(\xi \kappa), \kappa<\varepsilon<\eta}}\left(\boldsymbol{S}_{\kappa} \cdot \boldsymbol{S}_{\lambda}\right)\left(\boldsymbol{S}_{\varepsilon} \cdot \boldsymbol{S}_{\gamma}\right)\left(\boldsymbol{S}_{\eta} \cdot \boldsymbol{S}_{\xi}\right)+\frac{1}{32},
$$

$$
\begin{aligned}
\mathcal{P}_{a}^{M}= & \frac{7}{64}+\frac{7}{48} \sum_{i j \in\{\tilde{a}\}} \boldsymbol{S}_{i} \cdot \boldsymbol{S}_{j}+\frac{7}{60} \sum_{i j k l \in\{\tilde{a}\}}\left(\boldsymbol{S}_{i} \cdot \boldsymbol{S}_{j}\right)\left(\boldsymbol{S}_{k} \cdot \boldsymbol{S}_{l}\right) \\
& +\frac{1}{45} \sum_{i j k l m n \in\{\tilde{a}\}}\left(\boldsymbol{S}_{i} \cdot \boldsymbol{S}_{j}\right)\left(\boldsymbol{S}_{k} \cdot \boldsymbol{S}_{l}\right)\left(\boldsymbol{S}_{m} \cdot \boldsymbol{S}_{n}\right) .
\end{aligned}
$$

Here, $(i j),\langle i j\rangle$, and $\langle\langle i j\rangle\rangle$ represent, respectively, the nearest-, next-nearest-, and next-next-nearest-neighbor pairs, and $J_{1}$ $\gg J_{2}, J_{3}>0$. The summation $\Sigma_{\mu \nu} \cdots \in\{a\}$ is taken over sites in the ath hexagon. The operators $P_{4}^{a}$ and $P_{6}^{a}$ represent, respectively, the four-body and six-body ring exchange interactions, ${ }^{25}$

$$
\begin{gathered}
P_{4}^{a}=\frac{1}{4}+\sum_{\mu<\nu} \boldsymbol{S}_{\mu} \cdot \boldsymbol{S}_{\nu}+4 G_{i j k l}, \\
G_{i j k l}=\left(\boldsymbol{S}_{i} \cdot \boldsymbol{S}_{j}\right)\left(\boldsymbol{S}_{k} \cdot \boldsymbol{S}_{l}\right)+\left(\boldsymbol{S}_{i} \cdot \boldsymbol{S}_{l}\right)\left(\boldsymbol{S}_{j} \cdot \boldsymbol{S}_{k}\right)-\left(\boldsymbol{S}_{i} \cdot \boldsymbol{S}_{k}\right)\left(\boldsymbol{S}_{j} \cdot \boldsymbol{S}_{l}\right), \\
P_{6}^{a}=\frac{1}{16}+\frac{1}{4} \sum_{\mu<\nu} \boldsymbol{S}_{\mu} \cdot \boldsymbol{S}_{\nu}+\sum_{\mu<\mu<\eta<\epsilon} G_{\mu \nu \eta \epsilon}+4 S_{i j k l m n}, \\
S_{i j k l m n}=\sum_{C(i, j, k, l, m, n)}(-1)^{P}\left(\boldsymbol{S}_{\alpha} \cdot \boldsymbol{S}_{\beta}\right)\left(\boldsymbol{S}_{\gamma} \cdot \boldsymbol{S}_{\delta}\right)\left(\boldsymbol{S}_{\epsilon} \cdot \boldsymbol{S}_{\phi}\right),
\end{gathered}
$$

where the sum in Eq. (9) is taken over all combinations of $(i, j, k, l, m, n)$ in three different pairs depicted in Fig. 2. $(-1)^{P}$ is $1(-1)$ when a combination is generated by an even (odd) number of transpositions between different pairs. The summations $\Sigma_{A, B, C}^{a}$ are, respectively, taken over the four-spin configurations of types $A, B$, and $C$ of the $a$ th hexagon, as

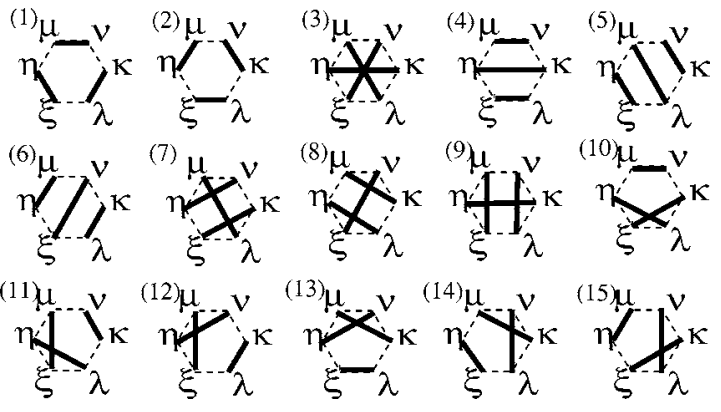

FIG. 2. The spin-singlet states of a hexagon.

depicted in Fig. 1(b). The operator $\mathcal{P}_{a}^{M}$ projects the six spins surrounding the $a$ th hexagon [depicted as $i, j, k, l, m, n$ in Fig. 1(a)] onto the subspace with total spin $S=3$. The summation $\Sigma_{i j} \cdots \in\{\widetilde{a}\}$ is taken over these six sites in the ath hexagon. $H_{\mathrm{K}}$ is the Hamiltonian of the $s=1 / 2$ Klein model on a honeycomb lattice, which was studied in detail by Chayes et $a l .26,27$ The ground-state space of $H_{\mathrm{K}}$ is spanned by valence bond (VB) states, which are formed from spin-singlet pairs of nearest-neighbor sites. Thus, all dimer covering states on the honeycomb lattice are macroscopically degenerate ground states of $H_{\mathrm{K}}$. An example of the dimer covering states is shown in Fig. 1(c). The spin-spin correlation functions for these VB states exhibit long-distance exponential decay, indicating the absence of magnetic long-range order. Also, the result of the single-mode approximation supports the existence of a spin excitation gap above the spin-singlet ground states. However, because each dimer state breaks the spatial symmetry of the system, a quantum spin liquid state is not the unique ground state of the Klein model.

In the following, we show that $H_{\mathrm{R}}$ introduces a resonance among these dimer covering states and, as a result, selects an almost unique ground state that can be expressed as a superposition of VB states, preserving the spatial symmetry. To this end, it is convenient to utilize the Schwinger boson representation for spin operators: ${ }^{28} S_{i}^{+}=u_{i}^{\dagger} d_{i}, S_{i}^{-}=d_{i}^{\dagger} u_{i}, S_{i}^{z}$ $=\left(u_{i}^{\dagger} u_{i}-d_{i}^{\dagger} d_{i}\right) / 2$, and $\hat{S}_{i}=\left(u_{i}^{\dagger} u_{i}+d_{i}^{\dagger} d_{i}\right) / 2$. Here the average value of $\hat{S}$ is $1 / 2$. We also introduce the spin-singlet operator $O_{i j} \equiv\left(u_{i} d_{j}-d_{i} u_{j}\right) / \sqrt{2}$. Then, each dimer state is expressed as

$$
|D\rangle=\prod_{(i j) \in \mathcal{D}} O_{i j}^{\dagger}|0\rangle .
$$

Here, $\mathcal{D}$ is the set of nearest-neighbor pairs corresponding to a particular realization of the dimer covering. We choose the phase convention of $O_{i j}$ as depicted in Fig. 1(c).

We now show that $H_{\mathrm{R}}$ is a variant of the QDM represented by spin operators. The Hamiltonian of the QDM is $^{18,22}$

$$
H_{\mathrm{QDM}}=-t[|1\rangle\langle 2|+| 2\rangle\langle 1|]+V[|1\rangle\langle 1|+| 2\rangle\langle 2|] .
$$

Here, $|1\rangle$ and $|2\rangle$ are the dimer covering states of a single hexagon corresponding, respectively, to the states (1) and (2) of Fig. 2. The first term in $H_{\mathrm{QDM}}$ is the kinetic term that transfers $|1\rangle$ to $|2\rangle$ and $|2\rangle$ to $|1\rangle$. The second term is the potential term. To construct operators that act as the first and 
second terms of $H_{\mathrm{QDM}}$ and are expressed in terms of spin operators, we consider all possible spin singlet states of a single hexagon, depicted in Fig. 2. Although the states except (1) and (2) in Fig. 2 are not the ground state of $H_{\mathrm{K}}$, the kinetic term for the states $|1\rangle$ and $|2\rangle$ are expressed by the linear combination of these states. To see this, we introduce the density operators for the singlet dimers on a hexagon corresponding to these 15 states,

$$
\begin{aligned}
& \mathcal{T}_{a}^{(1)}=O_{\nu \mu}^{\dagger} O_{\nu \mu} O_{\lambda \kappa}^{\dagger} O_{\lambda \kappa} O_{\eta \xi}^{\dagger} O_{\eta \xi}, \\
& \mathcal{T}_{a}^{(2)}=O_{\nu \kappa}^{\dagger} O_{\nu \kappa} O_{\lambda \xi}^{\dagger} O_{\lambda \xi} O_{\eta \mu}^{\dagger} O_{\eta \mu},
\end{aligned}
$$

and so forth. It is obvious that $\mathcal{T}^{(m)}|m\rangle=|m\rangle$ with $m$ $=1,2, \ldots, 15$. In particular, we are concerned with the action of $\mathcal{T}_{a}^{(1)}$ and $\mathcal{T}_{a}^{(2)}$ on the states $|1\rangle$ and $|2\rangle$, i.e.,

$$
\begin{gathered}
\mathcal{T}_{a}^{(1)}|1\rangle=|1\rangle, \quad \mathcal{T}_{a}^{(2)}|2\rangle=|2\rangle, \\
\mathcal{T}_{a}^{(1)}|2\rangle=-\frac{1}{4}|1\rangle, \quad \mathcal{T}_{a}^{(2)}|1\rangle=-\frac{1}{4}|2\rangle .
\end{gathered}
$$

The first pair of relations here implies that $\mathcal{T}_{a}^{(1)}$ and $\mathcal{T}_{a}^{(2)}$ act as the potential terms of $H_{\mathrm{QDM}}$. In the following, it is convenient to use the representations

$$
\mathcal{T}_{a}^{(1)}=h_{1 a}^{\dagger} h_{1 a}, \quad \mathcal{T}_{a}^{(2)}=h_{2 a}^{\dagger} h_{2 a},
$$

with

$$
\begin{aligned}
& h_{1 a}=O_{\nu \mu} O_{\lambda \kappa} O_{\eta \xi}, \\
& h_{2 a}=O_{\nu \kappa} O_{\lambda \xi} O_{\eta \mu} .
\end{aligned}
$$

Next, we introduce the operator

$$
\mathcal{K}_{a} \equiv-h_{1 a}^{\dagger} h_{2 a}-h_{2 a}^{\dagger} h_{1 a},
$$

which is (as can be seen by using the relations $O_{\mu \nu}^{\dagger} O_{\kappa \lambda}^{\dagger}$ $+O_{\mu \lambda}^{\dagger} O_{\nu \kappa}^{\dagger}=O_{\mu \kappa}^{\dagger} O_{\nu \lambda}^{\dagger}$, etc.) expressed as

$$
\mathcal{K}_{a}=-\frac{1}{2}\left(\sum_{m=1}^{2} \mathcal{T}_{a}^{(m)}-\mathcal{T}_{a}^{(3)}+\sum_{m=4}^{9} \mathcal{T}_{a}^{(m)}-\sum_{m=10}^{15} \mathcal{T}_{a}^{(m)}\right) .
$$

Note that $\mathcal{K}_{a}$ satisfies the following relations:

$$
\mathcal{K}_{a}|1\rangle=-|2\rangle+\frac{1}{4}|1\rangle, \quad \mathcal{K}_{a}|2\rangle=-|1\rangle+\frac{1}{4}|2\rangle .
$$

These actions of $\mathcal{K}_{a}$ are similar to those of the kinetic term of $H_{\mathrm{QDM}}$. To establish the complete set of relations between the QDM and the operators $\mathcal{K}_{a}, \mathcal{T}_{a}^{(1)}$, and $\mathcal{T}_{a}^{(2)}$, we need to verify their actions on unflippable dimers that are not flipped by $\mathcal{K}_{a}$, as shown in Fig. 1(c). It is easily shown that the actions of $\mathcal{K}$ and $\mathcal{T}^{(1)}+\mathcal{T}^{(2)}$ on the unflippable dimers generate dimer covering states that are not in the ground-state space of $H_{\mathrm{K}}$. These nonground states contain at least one singlet pair in the set of six spins surrounding the hexagon to which an operation is applied, i.e., the spins on $i, j, k, l, m, n$ in Fig. 1(a) when the operators are applied to the $a$ th hexagon. Thus, the unwanted states are excluded by the projection onto the maximum spin states of these six spins. This is carried out by applying the operator $\mathcal{P}_{a}^{M}$. Also, using the relation $\boldsymbol{S}_{i} \cdot \boldsymbol{S}_{j}=\frac{1}{4}$ $-O_{i j}^{\dagger} O_{i j}$, we find $\mathcal{K}_{a}=K_{a}, \mathcal{T}_{a}^{(1)}+\mathcal{T}_{a}^{(2)}=V_{a}$, thereby arriving at the Hamiltonian (2).

\section{B. Exact ground state}

We now show that the Hamiltonian $H=H_{\mathrm{K}}+H_{\mathrm{R}}$ has the same ground-state properties as the QDM in a certain parameter region. The lowest-energy sector in the Hilbert space of $H$ is spanned by VB states, provided that the ground state of $H_{\mathrm{R}}$ can be expressed as a linear combination of $|D\rangle$. To find the ground state, it is useful to rewrite $H_{\mathrm{R}}$ in the form

$$
\begin{aligned}
H_{\mathrm{R}}= & \frac{J_{2}+J_{3}}{2} \sum_{a} \mathcal{P}_{a}^{M}\left(h_{1 a}^{\dagger}-h_{2 a}^{\dagger}\right)\left(h_{1 a}-h_{2 a}\right)+\frac{J_{3}-J_{2}}{2} \\
& \times \sum_{a} \mathcal{P}_{a}^{M}\left(h_{1 a}^{\dagger}+h_{2 a}^{\dagger}\right)\left(h_{1 a}+h_{2 a}\right) .
\end{aligned}
$$

For $J_{3}>J_{2}$, the energy eigenvalues of $H_{\mathrm{R}}$ are nonnegative, and only unflippable dimer states are zero energy states. Therefore, the ground state of $H$ is a dimer covering without flippable dimers. If we simply impose periodic boundary conditions in both $x$ and $y$ directions, the ground state remains macroscopically degenerate. This degeneracy is removed by imposing periodic boundary conditions with a shift by one hexagon in the $y$ direction. Then, the unique ground state is the staggered VBC [see Fig. 1(d)], which was previously identified by Moessner et al. ${ }^{22}$

The energy levels of $H_{\mathrm{R}}$ are non-negative also in the case of $J_{2}=J_{3}$. Although the staggered VBC is obviously a zero energy state here too, in this case there also exists a nontrivial, liquidlike ground state. It is easily seen that the equalamplitude superposition of dimer states $|G\rangle \equiv \Sigma_{i}\left|D_{i}\right\rangle$ is the zero energy state of $H_{\mathrm{R}}$ if the summation $\Sigma_{i}$ is restricted within a sector of dimer states related by the local operations (14) and (20). Generally, a class of dimer states connected by these local operations is characterized by a topological number. ${ }^{18,29}$ On a honeycomb lattice, however, in addition to a topological number, the total number of dimers in each direction is also conserved by the local flip. Thus, $|G\rangle$ is the unique ground state of $H$ in a given sector specified by these conserved quantities. This state, preserving both the spinrotational and spatial symmetries, is a spin liquid state, and it is in the same universality class as the short-range RVB state. In fact, the upper limit of the spin-spin correlation function for this state exhibits long-distance exponential decay expressed by ${ }^{26}$

$$
\left|\left\langle G\left|S_{i}^{z} S_{j}^{z}\right| G\right\rangle\right| \leqslant(3 / 2) 2^{-\left|r_{i}-r_{j}\right|} .
$$

These results show that the QDM is realized as a spin system with multiple ring exchange interactions, and the case $J_{2}$ $=J_{3}$ corresponds to the Rokhsar-Kivelson (RK) point of the QDM. ${ }^{18}$

In the case of $J_{2}>J_{3}$, unfortunately, we have not been able to derive an exact ground state of $H$ analytically. How- 
ever, the plaquette VBC state obtained by Moessner et al. ${ }^{22}$ for the QDM in this parameter region is indeed an exact eigenstate of $H$, and it is possible that this is the ground state.

We now consider the Hamiltonian $H$ without the projection operator $\mathcal{P}_{a}^{M}$ in $H_{\mathrm{R}}$. Then, the model is more realistic, though its exact ground state is no longer accessible by analytical method. The operation of $K_{a}$ and $V_{a}$ on unflippable dimers does not vanish, but creates states which are not in the ground-state sector of $H_{\mathrm{K}}$. In this situation, the staggered VB solid state is not the zero-energy state even for $J_{3} \geqslant J_{2}$, and pushed up to a state with higher energy of order $J_{1}$. Thus, it is expected that, at the RK point $J_{2}=J_{3}$, the spin liquid state is the most plausible candidate for the true ground state provided that $J_{1} \gg J_{2}, J_{3}$.

\section{Dimer-dimer correlation in the spin liquid state}

In contrast to the magnetic correlation, the dimer correlation in the spin liquid state obeys a power law, as shown below. In the ground-state space, the dimer-dimer correlation is identical to that of the classical dimer model on a honeycomb lattice, which belongs to the universality class of the Gaussian model with central charge $c=1 .^{30}$ The correlation function for the dimer density operator at the bond $(i j), N_{i j}$ $=-\boldsymbol{S}_{i} \cdot \boldsymbol{S}_{j}+1 / 4$, displays the long-distance behavior

$$
\left\langle N_{i j} N_{l m}\right\rangle-\left\langle N_{i j}\right\rangle\left\langle N_{l m}\right\rangle \sim \frac{\left[\cos \left(4 \pi\left|r_{i}-r_{l}\right| / 3\right)-1\right]}{\left|r_{i}-r_{l}\right|^{2}},
$$

when the two dimers on $(i j)$ and $(l m)$ are in the same direction. ${ }^{30}$ This power-law decay implies that the lowenergy properties are governed by gapless nonmagnetic excitations. The low-lying excitation energy is computed using the single-mode approximation. We assume that the excited state takes the form $|k\rangle=\sum_{(i j)} e^{i k r_{i}} \delta N_{i j}|G\rangle$, with $\delta N_{i j}=N_{i j}$ $-\left\langle N_{i j}\right\rangle$. By definition, $|k\rangle$ is orthogonal to the ground state, i.e., $\langle k \mid G\rangle=0$. Therefore, the excitation energy is obtained from

$$
\varepsilon_{k}=\frac{\left\langle G\left|\left[\delta N_{-k}\left[H, \delta N_{k}\right]\right]\right| G\right\rangle}{\left\langle G\left|\delta N_{-k} \delta N_{k}\right| G\right\rangle} .
$$

The denominator of the right-hand side of this relation can be computed from the correlation function $\left\langle N_{i j} N_{k l}\right\rangle$ and behaves as $\sim \ln (R / a)$, where $a$ and $R$ are the lattice constant and the system size, respectively. When the excitation energy is sufficiently smaller than the spin excitation gap of $H_{\mathrm{K}}$, the total number of spin-singlet dimers is conserved, and also, $H_{\mathrm{K}}$ does not contribute to the low-lying excitations. Thus, the numerator of the right-hand side of the above relation for $\varepsilon_{k}$ reduces to $\sum_{i l}\left\langle G\left|\delta N_{i j} H_{\mathrm{R}} \delta N_{l m} r_{i} r_{l}\right| G\right\rangle k^{2}$ for small $k$. The coefficient of the $k^{2}$ term is expressed in terms of the three-body dimer correlation functions, which exhibit a leading logarithmic behavior of the form $\sim \ln (R / a)$. Hence, the logarithmic divergences in the denominator and numerator of $\varepsilon_{k}$ cancel out. We thus find that the gapless excitation energy in the spin-singlet sector is given by $\varepsilon_{k} \sim a k^{2}$. This dispersion relation is in accordance with Henley's conjecture based upon height representations. ${ }^{31}$

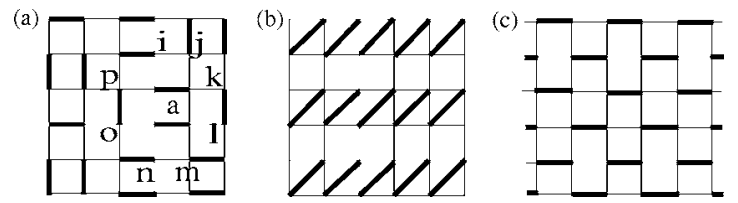

FIG. 3. (a) Dimer covering on a square lattice. (b) A ground state of $H_{\mathrm{K}}$ that is not a ground state of $H_{\mathrm{R}}$. (c) The staggered VB crystal.

\section{III. $s=1 / 2$ HEISENBERG ANTIFERROMAGNET ON A SQUARE LATTICE}

The analysis presented in the previous sections is easily extended to other lattice structures. For the model with $s$ $=1 / 2$ on the square lattice, the Hamiltonian is also given by $H=H_{\mathrm{K}}+H_{\mathrm{R}}$, but in this case we have the following:

$$
\begin{aligned}
& H_{\mathrm{K}}= J_{1}\left[\frac{1}{5} \sum_{(i j)} \boldsymbol{S}_{i} \cdot \boldsymbol{S}_{j}+\frac{1}{5} \sum_{\langle i j\rangle} \boldsymbol{S}_{i} \cdot \boldsymbol{S}_{j}+\frac{1}{5} \sum_{\langle\langle i j\rangle} \boldsymbol{S}_{i} \cdot \boldsymbol{S}_{j}\right. \\
&+\left.\frac{1}{40} \sum_{\substack{i \neq j, k \neq l, i \neq k, l \\
j \neq k, l}}\left(\boldsymbol{S}_{i} \cdot \boldsymbol{S}_{j}\right)\left(\boldsymbol{S}_{k} \cdot \boldsymbol{S}_{l}\right)+\frac{3}{64} N\right] \\
& H_{\mathrm{R}}=\sum_{a} \mathcal{P}_{a}^{M}\left[J_{2} K_{a}+J_{3} V_{a}\right], \\
& K_{a}=-\frac{1}{2} \sum_{(i j) \in\{a\}} \boldsymbol{S}_{i} \cdot \boldsymbol{S}_{j}+\frac{1}{4} P_{s 4}^{a}, \\
& V_{a}=\frac{1}{8}-\frac{1}{4} \sum_{(i j) \in\{a\}} S_{i} \cdot \boldsymbol{S}_{j}+\sum_{\substack{(i j)(k l) \in\{a\} \\
i<j<k<l}}\left(\boldsymbol{S}_{i} \cdot \boldsymbol{S}_{j}\right)\left(\boldsymbol{S}_{k} \cdot \boldsymbol{S}_{l}\right),
\end{aligned}
$$

$$
\mathcal{P}_{a}^{M}=-\frac{Q_{a}}{140}-\frac{Q_{a}^{2}}{280}+\frac{Q_{a}^{3}}{1260}+\frac{Q_{a}^{4}}{2520},
$$

with $Q_{a}=\Sigma_{i j \in\{a \tilde{a}\}} \boldsymbol{S}_{i} \cdot \boldsymbol{S}_{j}$. Here the summation $\Sigma_{i j \in\{\tilde{a}\}}$ is taken over the nearest-neighbor sites of the $a$ th square [the eight sites on $i, j, k, l, m, n, o, p$ in Fig. 3(a)]. The operator $P_{s 4}^{a}$ is the four-body ring exchange interaction on the $a$ th square. The ground-state space of the $s=1 / 2$ Klein model on a square lattice is more complicated than that of the model on a honeycomb lattice. In the case of a square lattice, in addition to dimer covering states, some states with spin-singlet pairs on next-nearest-neighbor sites are also in the groundstate space. An example of such states is depicted in Fig. 3(b). However, fortunately, such singlet states are not in the ground-state space of $H_{\mathrm{R}}$, and they can be excluded from our consideration. It is thus found that at the RK point $J_{2}=J_{3}$, under periodic boundary conditions in both $x$ and $y$ directions, the ground state of $H_{\mathrm{K}}+H_{\mathrm{R}}$ is the short-range RVB state $\Sigma_{D}|D\rangle$, where $|D\rangle$ is the dimer covering state on the square lattice. The low-energy properties of this short-range RVB state were investigated in detail by Rokhsar and 
(a)

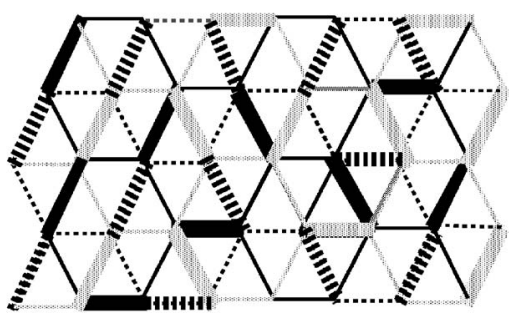

(b)

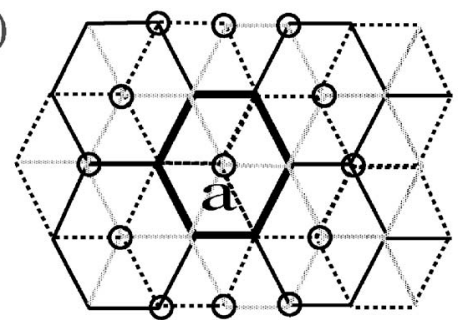

FIG. 4. (a) Triangular lattice divided into three honeycomb lattices, $h_{A}$ (black solid), $h_{B}$ (black dotted), and $h_{C}$ (gray). The bold lines represent an example of loop covering. (b) Open circles represent the nearest-neighbor sites of the $a$ th hexagon. The $a$ th hexagon is denoted by thick lines.

Kivelson. ${ }^{18}$ The dimer-dimer correlation obeys a opwer law, and the low-energy nonmagnetic excitation has the dispersion relation $\varepsilon_{k} \sim a k^{2} .{ }^{18}$ For $J_{3}>J_{2}$, the staggered VBC state depicted in Fig. 3(c) is the unique ground state.

\section{IV. $s=1$ HEISENBERG ANTIFERROMAGNET ON A TRIANGULAR LATTICE}

\section{A. Model and the exact ground state}

In this case, we divide the triangular lattice into three honeycomb lattices, $h_{A}, h_{B}$, and $h_{C}$, as shown in Fig. 4(a). Here, each site consists of two sites of two different honeycomb lattices. The Hamiltonian on this lattice is also given by $H=H_{\mathrm{K}}+H_{\mathrm{R}}$. The Klein Hamiltonian $H_{\mathrm{K}}$ for this system has the same form as that given in Eq. (1), but here it is defined on a triangular lattice, and it is expressed in terms of the $s=1$ spin operators. The ground-state space of this Klein model is spanned by states fully packed with loops composed of singlet dimers on the three kinds of hexagons arranged sequentially in the order $A, B, C, A, B, C, \ldots$. We show an example of loop covering states in Fig. 4. Resonance among these loop covering states is introduced as

$$
H_{\mathrm{R}}=H_{A}+H_{B}+H_{C},
$$

where $H_{A}, H_{B}$, and $H_{C}$ are defined on $h_{A}, h_{B}$, and $h_{C}$, respectively, and take the form of Eq. (2) with $\mathcal{P}_{a}^{M}$ replaced by the projection onto the maximum spin state of the 13 nearestneighbor spins of the ath hexagon on the parent triangular lattice,

$$
\mathcal{P}_{a}^{M}=\prod_{s=0}^{12} \frac{\left(\sum_{i \in\{\tilde{a}\}} S_{i}\right)^{2}-s(s+1)}{182-s(s+1)} .
$$

Here the summation $\Sigma_{i \in\{\tilde{a}\}}$ is taken over the nearest-neighbor sites of the $a$ th hexagon. [See Fig. 4(b).]

The ground-state properties are similar to the models considered in the previous sections. At the RK point, the equalamplitude superposition of loop covering states is the unique ground state in a given sector. The loop statistics of this state are described by a classical loop model referred to as the "red-green-blue model." This ground state represents a new universality class of a spin liquid described by the quantum loop model.

\section{B. Dimer-dimer correlation in the spin liquid state}

As mentioned in the previous section, in the spin liquid state realized at the RK point for the $s=1$ triangular model, the loop-loop correlation is described by the red-green-blue model, which is constructed from three honeycomb lattices as explained in Sec. IV A, and belongs to the universality class of the central charge $c=1+1+1=3$, i.e., the sum of three mutually independent Gaussian models. For this loop model, the local density of loops is equivalent to the local dimer density in one of the three honeycomb-lattice dimer models. Since the three honeycomb-lattice dimer models do not couple with each other, the loop correlation function of the triangular model is nothing but the dimer correlation function of the honeycomb-lattice-dimer model, and exhibits power-law long-distance behavior given by Eq. (23). ${ }^{32}$ Then, applying the single-mode approximation as discussed in Sec. III C, we find that the low-energy excitation energy in the spin singlet-singlet channel is gapless and its dispersion relation is given by $\varepsilon_{k} \propto k^{2}$.

\section{DISCUSSION AND SUMMARY}

In this paper, we have presented rigorous results for twodimensional quantum spin systems with multiple ring exchange interactions that possess quantum spin liquid ground states. It should be noted that Misguich et al. and LiMing et $a l$. showed that for the $s=1 / 2$ Heisenberg antiferromagnet on a triangular lattice, sufficiently strong ring exchange interactions destroy magnetic long-range order, and stabilize a spin liquid state. ${ }^{16,17}$ The spin liquid state found by them has a remarkable similarity with the ground state discussed in the present paper: exponentially decaying spin-spin correlations, and a large number of spin singlet excitations in the spin gap (at least for some parameter regions). Since the classical Heisenberg model with ring exchange interactions does not possess macroscopic degeneracy, we speculate that the underlying structure of the model may have a close connection with the Klein model, which is the origin of singlet gapless excitations in our models.

These observations imply that the existence of strong ring exchange interactions in addition to geometrical frustration generically supports the realization of the spin liquid in quantum antiferromagnets, though the models considered in the present paper are rather complicated. The multiple ring exchange interaction is a result of strong quantum fluctuations, which destabilize conventional magnetic orders. In real systems, the effects of such strong quantum fluctuations have 
been extensively studied so far in connection with the magnetism of solid ${ }^{3} \mathrm{He} .{ }^{25}$ Also, it has been discussed that multiple ring exchange interactions may affect significantly the magnetic structures of $\mathrm{NiS}_{2} \cdot{ }^{33}$ Thus, there is a possibility that the quantum spin liquid state due to ring exchange interactions may be realized in real systems with sufficiently strong multiple ring exchange interactions.

\section{ACKNOWLEDGEMENTS}

The author is grateful to S. Miyashita and M. Oshikawa for valuable discussions. This work was partly supported by a Grant-in-Aid from the Ministry of Education, Science, Sports and Culture, Japan.
${ }^{1}$ P. W. Anderson, Mater. Res. Bull. 8, 153 (1973); P. Fazekas and P. W. Anderson, Philos. Mag. 30, 432 (1974).

${ }^{2}$ B. Canals and C. Lacroix, Phys. Rev. Lett. 80, 2933 (1998).

${ }^{3}$ M. Isoda and S. Mori, J. Phys. Soc. Jpn. 67, 4022 (1998).

${ }^{4}$ R. Moessner and J. T. Chalker, Phys. Rev. Lett. 80, 2929 (1998).

${ }^{5}$ Y. Yamashita and K. Ueda, Phys. Rev. Lett. 85, 4960 (2000).

${ }^{6}$ A. Koga and N. Kawakami, Phys. Rev. B 63, 144432 (2001).

${ }^{7}$ H. Tsunetsugu, J. Phys. Soc. Jpn. 70, 640 (2001).

${ }^{8}$ S. Fujimoto, Phys. Rev. Lett. 89, 226402 (2002); Phys. Rev. B 67, 235102 (2003).

${ }^{9}$ J. T. Chalker, P. C. W. Holdsworth and E. F. Shender, Phys. Rev. Lett. 68, 855 (1992).

${ }^{10}$ S. Sachdev, Phys. Rev. B 45, 12377 (1992).

${ }^{11}$ J. von Delft and C. L. Henley, Phys. Rev. Lett. 69, 3236 (1992).

${ }^{12}$ I. Ritchey, P. Chandra, and P. Coleman, Phys. Rev. B 47, 15342 (1993).

${ }^{13}$ P. Lecheminant, B. Bernu, C. Lhuillier, L. Pierre, and P. Sindzingre, Phys. Rev. B 56, 2521 (1997).

${ }^{14}$ F. Mila, Phys. Rev. Lett. 81, 2356 (1998).

${ }^{15}$ C. Zeng and V. Elser, Phys. Rev. B 42, 8436 (1990); 51, 8318 (1995)

${ }^{16}$ G. Misguich, B. Bernu, C. Lhuillier, and C. Waldtmann, Phys. Rev. Lett. 81, 1098 (1998); M. Misguich, C. Lhuillier, B. Bernu, and C. Waldtmann, Phys. Rev. B 60, 1064 (1999).

${ }^{17}$ W. LiMing, G. Misguich, P. Sindzinger, and C. Lhuillier, Phys. Rev. B 62, 6372 (2000).

${ }^{18}$ D. S. Rokhsar and S. A. Kivelson, Phys. Rev. Lett. 61, 2376
(1988).

${ }^{19}$ B. Sutherland, Phys. Rev. B 37, R3786 (1988).

${ }^{20}$ M. Kohmoto and Y. Shapir, Phys. Rev. B 37, 9439 (1988).

${ }^{21}$ R. Moessner and S. L. Sondhi, Phys. Rev. Lett. 86, 1881 (2001).

${ }^{22}$ R. Moessner, S. L. Sondhi, and P. Chandra, Phys. Rev. B 64, 144416 (2001).

${ }^{23}$ G. Misguich, D. Serban, and V. Pasquier, Phys. Rev. Lett. 89, 137202 (2002); 67, 214413 (2003).

${ }^{24} \mathrm{~A}$ correspondence between the QDM and spin systems with multiple exchange interactions that is quite different from the correspondence considered in the present paper is studied in L. Balents, M. P. A. Fisher, and S. M. Girvin, Phys. Rev. B 65, 224412 (2002); C. Nayak and K. Shtengel, ibid. 64, 064422 (2001).

${ }^{25}$ H. Godfrin and D. D. Osheroff, Phys. Rev. B 38, 4492 (1988).

${ }^{26}$ D. J. Klein, J. Phys. A 15, 661 (1982).

${ }^{27}$ J. T. Chayes, L. Chayes, and S. A. Kivelson, Commun. Math. Phys. 123, 53 (1989).

${ }^{28}$ See, e.g., D. P. Arovas, A. Auerbach, and F. D. M. Haldane, Phys. Rev. Lett. 60, 531 (1988).

${ }^{29}$ N. E. Bonesteel, Phys. Rev. B 40, 8954 (1989).

${ }^{30}$ B. Nienhuis, H. J. Hilhorst, and H. W. J. Blöte, J. Phys. A 17, 3559 (1984).

${ }^{31}$ C. L. Henley, J. Phys.: Condens. Matter 16, S891 (2004).

${ }^{32}$ D. Das and J. L. Jacobsen, J. Phys. A 37, 2003 (2004).

${ }^{33}$ K. Yosida and S. Inagaki, J. Phys. Soc. Jpn. 50, 3268 (1981). 